\title{
Effects of $\alpha$-tocopherol and $\beta$-carotene supplementation on liver cancer incidence and chronic liver disease mortality in the ATBC study
}

\begin{abstract}
G Y Lai ${ }^{\star}, 1,2$, S J Weinstein ${ }^{2}$, P R Taylor ${ }^{3}$, K A McGlynn ${ }^{4}$, J Virtamo ${ }^{5}$, M H Gail ${ }^{6}$, D Albanes ${ }^{2}$ and N D Freedman ${ }^{2}$
${ }^{1}$ Cancer Prevention Fellowship Program, National Cancer Institute, 9609 Medical Center Drive, Rm. 2W136, MSC 9712, Bethesda, MD 20892, USA; ${ }^{2}$ Nutritional Epidemiology Branch, Division of Cancer Epidemiology and Genetics, National Cancer Institute, 9609 Medical Center Drive, Bethesda, MD 20892, USA; ${ }^{3}$ Genetic Epidemiology Branch, Division of Cancer Epidemiology and Genetics, National Cancer Institute, 9609 Medical Center Drive, Bethesda, MD 20892, USA; ${ }^{4}$ Hormonal and Reproductive Epidemiology Branch, Division of Cancer Epidemiology and Genetics, National Cancer Institute, 9609 Medical Center Drive, Bethesda, MD 20892, USA; ${ }^{5}$ Department of Chronic Disease Prevention, National Institute for Health and Welfare, Fl-00271 Helsinki, Finland and 'Biostatistics Branch, Division of Cancer Epidemiology and Genetics, National Cancer Institute, 9609 Medical Center Drive, Bethesda, MD 20892, USA
\end{abstract}

Background: Recent data suggest the possible benefits of $\alpha$-tocopherol and $\beta$-carotene supplementation on liver cancer and chronic liver disease (CLD), but the long-term trial data are limited.

Methods: We evaluated the efficacy of supplemental $50 \mathrm{mg} \mathrm{day}^{-1} \alpha$-tocopherol and $20 \mathrm{mg}$ day ${ }^{-1} \beta$-carotene on incident liver cancer and CLD mortality in a randomised trial of 29105 Finnish male smokers, who received supplementation for 5-8 years and were followed for 16 additional years for outcomes.

Results: Supplemental $\alpha$-tocopherol, $\beta$-carotene, or both, relative to placebo, did not reduce the risk of liver cancer or CLD, either overall, during the intervention or during the post-intervention period.

Conclusions: Long-term supplemental $\alpha$-tocopherol or $\beta$-carotene had no effect on liver cancer or CLD mortality over 24 years of follow-up.

As inflammation and oxidative stress are thought to be important causes of liver cancer and liver disease (Poli, 2000; Luedde and Schwabe, 2011), antioxidants might have a beneficial effect. Several previous observational studies have indicated that higher serum levels of serum $\alpha$-tocopherol, $\beta$-carotene, and retinol are associated with a reduced risk of liver cancer or disease (Pan et al, 1993; Yu et al, 1995; Yamamoto et al, 1998; Yu et al, 1999; Clemente et al, 2002; Ito et al, 2006; Yuan et al, 2006). Other studies, including trials, have reported beneficial effects of vitamin $\mathrm{E}$ supplementation on these outcomes (Sanyal et al,
2004; Yakaryilmaz et al, 2007; Bjelakovic et al, 2011; Zhang et al, 2012) including recent data on non-diabetic persons with NASH (non-alcoholic steatohepatitis) in the PIVENS (Pioglitazone versus Vitamin E versus Placebo for the Treatment of Nondiabetic Patients with Nonalcoholic Steatohepatitis) trial (Sanyal et al, 2010) that has served as the basis of recent AASLD clinical recommendations (Chalasani et al, 2012). Concordant results were recently found for the use of vitamin E supplements and liver cancer in an observational cohort in Shanghai, China (Zhang et al, 2012). However, the long-term effect of vitamin $\mathrm{E}$ 
supplementation on liver disease is unclear (Lirussi et al, 2007; Musso et al, 2010; Bjelakovic et al, 2011).

\section{MATERIALS AND METHODS}

We evaluated the effects of $\alpha$-tocopherol and $\beta$-carotene supplementation on liver cancer incidence and chronic liver disease (CLD) mortality in the Alpha-Tocopherol Beta-Carotene Cancer Prevention (ATBC) study, a large randomised placebocontrolled trial of 29105 Finnish male smokers, aged 50-69 at baseline, without self-reported cirrhosis who received $50 \mathrm{mg}^{-1} \mathrm{day}^{-1}$ DL- $\alpha$-tocopheryl acetate, $20 \mathrm{mg} \mathrm{day}^{-1} \beta$-carotene, both, or placebo from enrolment in 1985-1988 until April 30, 1993 (The ATBC Cancer Prevention Study Group, 1994). Follow-up was through cancer diagnosis, death, or until 31 December 2009. Although designed to examine lung cancer, ATBC provided a unique opportunity to evaluate the long-term effects of $\alpha$-tocopherol and $\beta$-carotene supplementation on liver cancer incidence and CLD mortality.

Incident liver cancer cases $(n=208$; ICD-9 $=155$, and ICD$10=\mathrm{C} 22)$ and mortality from $\operatorname{CLD}(n=237$; ICD-9 $=571$, and ICD-10 $=\mathrm{K} 70, \mathrm{~K} 73$, or K74) were identified through the Finnish Cancer Registry (Korhonen et al, 2002) and Finnish Register of Causes of Death, respectively. All statistical analyses were based on intention-to-treat. Kaplan-Meier survival curves were plotted for the four intervention groups on the scale of time since randomisation. The effect of intervention was estimated by Cox proportional hazards regression with age as the time scale and allowing for left truncation.

We examined the results in the pre-specified baseline subgroups, defined by age, alcohol use, BMI, cigarettes per day, baseline serum $\alpha$-tocopherol, serum $\beta$-carotene, or serum total cholesterol. These analyses were designed primarily to assess consistency of the overall results, rather than discover subsets where treatment effects might be present. We also assessed associations among a subset of participants with measured hepatitis $\mathrm{B}(\mathrm{HBV})$ and $\mathrm{C}(\mathrm{HCV})$ viral status, and fasting glucose and insulin. Diabetes status was defined as: without diabetes (blood glucose $<100 \mathrm{mg} \mathrm{dl}^{-1}$ and no history of diabetes), prediabetes (blood glucose between 100 and $<126 \mathrm{mg} \mathrm{dl}^{-1}$ and no history of diabetes), and diabetes (blood glucose $\geqslant 126 \mathrm{mg} \mathrm{dl}^{-1}$ or selfreported history of diabetes). This subset analysis was motivated by findings in the literature suggesting a benefit from $\alpha$-tocopherol in the absence of diabetes. We used a $P$-value of 0.05 for statistical significance and all the tests were two-sided. Additional details on the methods can be found in the supplement.

\section{RESULTS}

The ATBC trial was randomised, and the baseline characteristics were similar across each intervention arm (Supplementary Table 1). During follow-up, 208 incident liver cancer cases and 237 deaths from CLD occurred. We observed no effect of supplemental $\alpha$-tocopherol, $\beta$-carotene, or both, relative to placebo, on liver cancer incidence (hazard ratio (HR) for $\alpha$-tocopherol, 1.18; $95 \%$ confidence interval (CI), 0.79-1.75; HR for $\beta$-carotene, 1.26 ; $95 \% \mathrm{CI}$, $0.85-1.87$; HR for both, $1.21,95 \%$ CI, $0.81-1.80$ ) or CLD mortality (HR for $\alpha$-tocopherol, 0.97; 95\% CI, $0.68-1.40$; HR for $\beta$-carotene, 1.06; 95\% CI, 0.74-1.51; HR for both, 1.01, 95\% CI, 0.70-1.45). Similar results were found during the intervention and postintervention period (Table 1; Figures 1 and 2).

Results were similar across the examined subgroups previously mentioned in the methods section (data not shown) and after excluding the few $(0.8 \%$ for HBsAg) HBV or ( $1.6 \%$ for anti-HCV)
HCV-positive cases (data not shown), although they appeared to vary by diabetes status. Among a subset of participants with information on fasting insulin and glucose, an effect of $\alpha$-tocopherol vs. no $\alpha$-tocopherol on CLD was observed among men without diabetes (odds ratio (OR), 0.53; 95\% CI, 0.33-0.86), but not among men with prediabetes (OR, 1.53; 95\% CI, 0.94-2.47) or diabetes (OR, 1.27; 95\% CI, 0.51-3.14; $P$ interaction $=0.009$ ) (Table 1). A suggestive protective effect was observed in $\beta$-carotene vs. no $\beta$-carotene on CLD in men with diabetes (OR, 0.46; $95 \% \mathrm{CI}$, $0.18-1.17)$ but not in men without diabetes or with prediabetes $(P$ interaction $=0.09)$. No differences by diabetes status were observed for liver cancer (all Pinteractions >0.20) (Table 1).

\section{DISCUSSION}

Overall, our study provides no evidence for a benefit of supplementation with $\alpha$-tocopherol and/or $\beta$-carotene supplementation on liver cancer incidence or CLD mortality in middle-aged participants. Indeed, the observed HRs exceeded 1.00 for most comparisons. It is possible that intervention benefits were not detected because the numbers of incident liver cancers and deaths from CLD were limited. The lower confidence limits in the analyses do suggest that if there are risk reductions from these interventions that we could not detect because of limited sample size, they would be modest $(21 \%$ and $32 \%$ lower from supplemental $\alpha$-tocopherol for liver cancer and CLD mortality, respectively, and $15 \%$ and $26 \%$ lower from supplemental $\beta$-carotene).We also note that our subgroup analyses (data not shown) indicated consistent null findings across a range of other exposures.

An exception to this pattern was evidence for a benefit of $\alpha$-tocopherol on CLD mortality in men without diabetes. It is not clear why the effects may differ by diabetes, and chance is a possible explanation. Although these results are consistent with recent findings from the PIVENS trial (Sanyal et al, 2010), which observed that daily supplementation with $800 \mathrm{IU}$ of $\alpha$-tocopherol had a beneficial effect on NASH progression over 96 weeks in patients lacking diabetes, there are substantial differences between the design of PIVENS and ATBC. A lower dose of $\alpha$-tocopherol was given in our study (50 IU) and our supplementation period (5-8 years) and follow-up (24 years) were substantially longer. Also, the PIVENS trial enrolled participants with pre-existing $\mathrm{NASH}$, whereas the ATBC study enrolled participants from the general population, most of who probably lacked NASH. Several other smaller trials have reported a beneficial effect of vitamin $\mathrm{E}$ supplementation on treating patients with NASH (Sanyal et al, 2004; Yakaryilmaz et al, 2007; Bjelakovic et al, 2011) and for other liver diseases (Bjelakovic et al, 2011). These studies, like the PIVENS trial, enrolled a specific population, that is, patients with pre-existing liver disease and supplemented with higher doses of vitamin E, which contrast notably with the general Finnish population that made up the ATBC study participants and the lower dose of $\alpha$-tocopherol.

Our analyses treated deaths from other causes as independent censoring at the time of the death. Unlike analyses of cumulative incidence over a long time interval (Gray, 1988), these analyses would not be distorted by differences in the amount of competing mortality among the intervention groups. In any case, mortality rates from other causes were similar across the treatment groups, both overall and in participants with and without diabetes.

Other data on micronutrient supplementation and liver cancer are sparse. Whereas a Chinese observational cohort recently reported an inverse association of both vitamin E supplement use and dietary vitamin $\mathrm{E}$ intake with liver cancer (Zhang et al, 2012), no effect was observed for 'factor $D$ ' $(\beta$-carotene $(15 \mathrm{mg})$, 
Table 1. Effect of $\alpha$-tocopherol and $\beta$-carotene on incident liver cancer and chronic liver disease mortality, ATBC Study

\begin{tabular}{|c|c|c|c|c|c|c|c|c|}
\hline & Placebo & $\alpha$-Tocopherol & $\beta$-Carotene & $\begin{array}{c}\alpha \text {-Tocopherol }+ \\
\beta \text {-carotene }\end{array}$ & $\begin{array}{c}\text { No } \\
\alpha \text {-tocopherol }\end{array}$ & $\alpha$-Tocopherol & $\begin{array}{c}\text { No } \\
\beta \text {-carotene }\end{array}$ & $\beta$-Carotene \\
\hline \multicolumn{9}{|c|}{ Incident liver cancer } \\
\hline \multicolumn{9}{|l|}{ All participants ${ }^{a}$} \\
\hline Entire period & 1.00 & $1.18(0.79-1.75)$ & $1.26(0.85-1.87)$ & $1.21(0.81-1.80)$ & 1.00 & $1.06(0.81-1.39)$ & 1.00 & $1.13(0.86-1.49)$ \\
\hline Cases/all & $10 / 7282$ & $9 / 7280$ & $8 / 7274$ & 9/7269 & $18 / 14556$ & $18 / 14549$ & $19 / 14562$ & $17 / 14543$ \\
\hline Trial period & 1.00 & $0.88(0.36-2.17)$ & $0.76(0.30-1.93)$ & $0.88(0.36-2.15)$ & 1.00 & $1.00(0.52-1.92)$ & 1.00 & $0.87(0.45-1.68)$ \\
\hline Cases/all & $35 / 6433$ & $44 / 6412$ & $48 / 6360$ & $45 / 6339$ & $83 / 12793$ & $89 / 12751$ & $79 / 12845$ & $93 / 12699$ \\
\hline \multicolumn{9}{|l|}{ Subset $^{b}$} \\
\hline Cases/controls & $10 / 98$ & $14 / 107$ & $10 / 83$ & $11 / 78$ & 20/181 & $25 / 185$ & $24 / 205$ & $21 / 161$ \\
\hline Without diabetes & 1.00 & $1.38(0.58-3.32)$ & $1.14(0.44-2.93)$ & $1.46(0.58-3.69)$ & 1.00 & $1.33(0.70-2.52)$ & 1.00 & $1.08(0.57-2.03)$ \\
\hline Cases/controls & $18 / 67$ & $18 / 79$ & $19 / 75$ & $14 / 72$ & $37 / 142$ & $32 / 151$ & $36 / 146$ & $33 / 147$ \\
\hline Prediabetes & 1.00 & $0.91(0.43-1.93)$ & $0.99(0.47-2.07)$ & $0.70(0.32-1.55)$ & 1.00 & $0.81(0.48-1.39)$ & 1.00 & $0.89(0.52-1.52)$ \\
\hline Cases/controls & $6 / 16$ & $5 / 11$ & $7 / 23$ & $12 / 12$ & $13 / 39$ & $17 / 23$ & $11 / 27$ & $19 / 35$ \\
\hline With diabetes & 1.00 & $0.94(0.21-4.13)$ & $0.92(0.25-3.45)$ & $3.08(0.84-11.29)$ & 1.00 & $2.03(0.81-5.09)$ & 1.00 & $1.70(0.65-4.45)$ \\
\hline Cases/all & $14 / 7282$ & $18 / 7280$ & $16 / 7274$ & $20 / 7269$ & $30 / 14556$ & $38 / 14549$ & $32 / 14562$ & $36 / 14543$ \\
\hline Trial period & 1.00 & $1.27(0.63-2.56)$ & $1.12(0.55-2.30)$ & $1.40(0.71-2.78)$ & 1.00 & $1.26(0.78-2.03)$ & 1.00 & $1.11(0.69-1.79)$ \\
\hline Cases/all & $45 / 6433$ & $39 / 6412$ & $46 / 6360$ & $39 / 6339$ & $91 / 12793$ & $78 / 12751$ & $84 / 12845$ & $85 / 12699$ \\
\hline Post-trial period & 1.00 & $0.87(0.57-1.33)$ & $1.04(0.69-1.56)$ & $0.88(0.57-1.35)$ & 1.00 & $0.86(0.63-1.16)$ & 1.00 & $1.09(0.81-1.47)$ \\
\hline \multicolumn{9}{|l|}{ Subset $^{b}$} \\
\hline Cases/controls & $31 / 98$ & $14 / 107$ & $27 / 83$ & $19 / 78$ & $58 / 181$ & $33 / 185$ & $45 / 205$ & $46 / 161$ \\
\hline Without diabetes & 1.00 & $0.40(0.20-0.80)$ & $1.01(0.55-1.83)$ & $0.71(0.37-1.36)$ & 1.00 & $0.53(0.33-0.86)$ & 1.00 & $1.26(0.79-2.00)$ \\
\hline Cases/controls & $14 / 67$ & $27 / 79$ & $21 / 75$ & $30 / 72$ & $35 / 142$ & $57 / 151$ & $41 / 146$ & $51 / 147$ \\
\hline Prediabetes & 1.00 & $1.51(0.72-3.13)$ & $1.23(0.57-2.63)$ & $1.96(0.95-4.04)$ & 1.00 & $1.53(0.94-2.47)$ & 1.00 & $1.23(0.77-1.98)$ \\
\hline Cases / controls & $10 / 16$ & 9/11 & $8 / 23$ & $4 / 12$ & $18 / 39$ & $13 / 23$ & $19 / 27$ & $12 / 35$ \\
\hline With diabetes & 1.00 & $1.59(0.46-5.48)$ & $0.60(0.18-1.96)$ & $0.49(0.11-2.09)$ & 1.00 & $1.27(0.51-3.14)$ & 1.00 & $0.46(0.18-1.17)$ \\
\hline
\end{tabular}

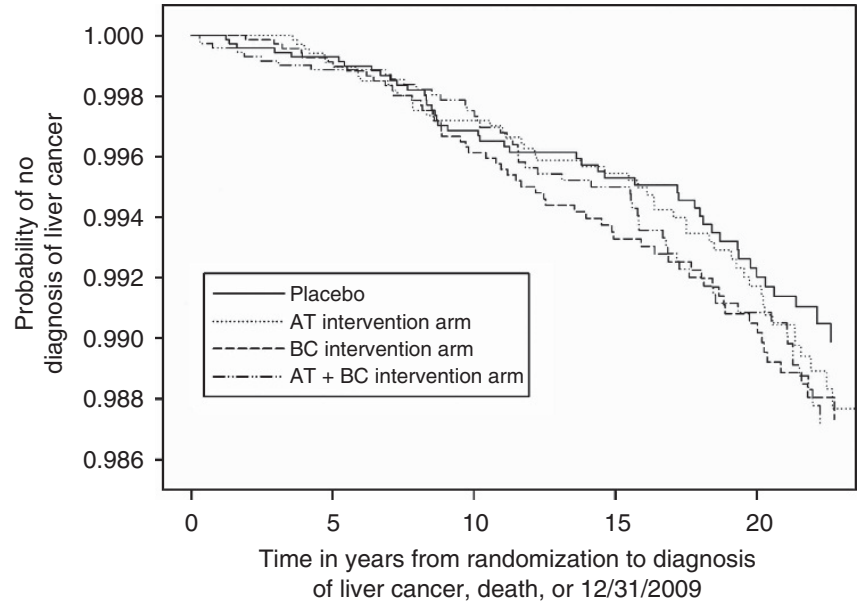

Figure 1. Kaplan-Meier estimates of the probability of no diagnosis of liver cancer among participants randomised to $\alpha$-tocopherol (AT), $\beta$-carotene $(\mathrm{BC})$, combination or placebo arms.

$\alpha$-tocopherol $(30 \mathrm{mg})$, and selenium $(50 \mu \mathrm{g}))$ on liver cancer mortality, relative to placebo in the Linxian General Population Nutrition Intervention Trial (Qu et al, 2007).

In apparent contrast with our results for $\alpha$-tocopherol and $\beta$-carotene supplementation, micronutrient status, as assessed by serum levels, has been associated with lower risk of liver cancer in several prospective cohort studies (Knekt et al, 1991; Yu et al, 1995; Ito et al, 2006; Yuan et al, 2006), including in the current one (companion paper). Within ATBC, we observed that higher baseline levels of serum $\beta$-carotene and retinol were associated

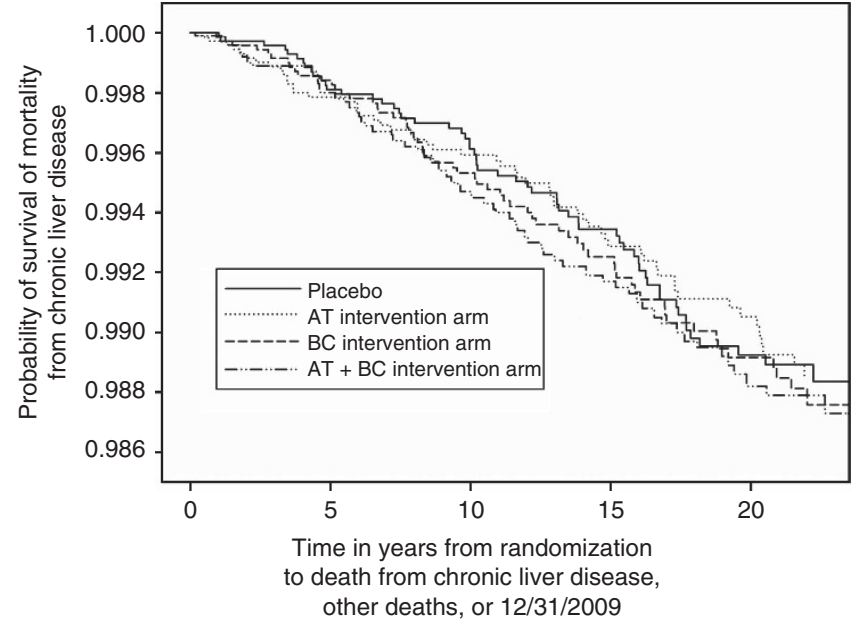

Figure 2. Kaplan-Meier estimates of the probability of survival from mortality from chronic liver disease among participants randomised to $\alpha$-tocopherol (AT), $\beta$-carotene (BC), combination or placebo arms.

with a reduced risk of incident liver cancer and CLD mortality, whereas higher baseline $\alpha$-tocopherol levels were associated with a lower risk of CLD mortality but not incident liver cancer. Such contrasting results do not necessarily conflict. Serum micronutrient status reflects on the long-term dietary intake and metabolism. Micronutrient supplementation during middle age may miss the important window of liver disease and liver cancer development. Alternatively, serum micronutrient levels may serve as a proxy for another aspect of diet or lifestyle, and may not actually have a direct role in the development of liver disease and liver cancer. 
Our study has several strengths, including large size, randomised design, evaluation of $\alpha$-tocopherol or $\beta$-carotene supplementation separately, long-term follow-up over 24 years, minimal loss to follow-up, and information on major liver cancer risk factors including HBV, HCV, alcohol, and diabetes. Limitations included the modest numbers of liver cancer cases and deaths from CLD; the primary purpose of the ATBC Study was to evaluate whether these supplements could reduce the lung cancer risk. We lacked information about fibrosis and NASH at baseline and also about incident liver disease. However, ATBC excluded subjects with a baseline history of cirrhosis or alcoholism. As ATBC was conducted among Finnish male smokers, our results may not apply to other populations. Finally, liver cancer incidence and mortality from CLD were not primary end points of ATBC, and we examined numerous subgroups. Thus findings in subgroups may well be due to chance.

In summary, in a large randomised trial, $\alpha$-tocopherol and $\beta$-carotene supplementation had no overall effect on the incidence of liver cancer or on mortality from CLD over the course of 24 years of follow-up either during the intervention period or in the post-intervention follow-up period. Effects were noted in certain subgroups of baseline diabetes status, but these findings were based on a small number of cases. Future studies are needed to examine these subgroup findings, as they may be due to chance.

\section{ACKNOWLEDGEMENTS}

We thank all individuals for their participation in this study. This research was supported by the Intramural Research Program, Division of Cancer Epidemiology and Genetics, National Cancer Institute, National Institutes of Health, Department of Health and Human Services and US Public Health Service contracts (N01-CN45165, N01-RC-45035, and N01-RC-37004). The ATBC Study was registered with clinicaltrials.gov (\#NCT00342992).

\section{CONFLICT OF INTEREST}

The authors declare no conflict of interest.

\section{DISCLAIMER}

The sponsor reviewed and approved final submission but had no role in the design and conduct of the study; collection, management, analysis and interpretation of the data; and preparation of the manuscript.

\section{REFERENCES}

Bjelakovic G, Gluud LL, Nikolova D, Bjelakovic M, Nagorni A, Gluud C (2011) Antioxidant supplements for liver diseases. Cochrane Database Syst Rev 3: CD007749.

Chalasani N, Younossi Z, Lavine JE, Diehl AM, Brunt EM, Cusi K, Charlton M, Sanyal AJ (2012) The diagnosis and management of non-alcoholic fatty liver disease: practice Guideline by the American Association for the Study of Liver Diseases, American College of Gastroenterology, and the American Gastroenterological Association. Hepatology 55(6): 2005-2023.

Clemente C, Elba S, Buongiorno G, Berloco P, Guerra V, Di Leo A (2002) Serum retinol and risk of hepatocellular carcinoma in patients with child-Pugh class A cirrhosis. Cancer Lett 178(2): 123-129.
Gray RJ (1988) A Class of K-Sample Tests for Comparing the Cumulative Incidence of a Competing Risk. Ann Stat 16(3): 1141-1154.

Ito Y, Suzuki K, Ishii J, Hishida H, Tamakoshi A, Hamajima N, Aoki K (2006) A population-based follow-up study on mortality from cancer or cardiovascular disease and serum carotenoids, retinol and tocopherols in Japanese inhabitants. Asian Pac J Cancer Prev 7(4): 533-546.

Knekt P, Aromaa A, Maatela J, Alfthan G, Aaran RK, Nikkari T, Hakama M, Hakulinen T, Teppo L (1991) Serum micronutrients and risk of cancers of low incidence in Finland. Am J Epidemiol 134(4): 356-361.

Korhonen P, Malila N, Pukkala E, Teppo L, Albanes D, Virtamo J (2002) The Finnish Cancer Registry as follow-up source of a large trial cohort-accuracy and delay. Acta Oncol 41(4): 381-388.

Lirussi F, Azzalini L, Orando S, Orlando R, Angelico F (2007) Antioxidant supplements for non-alcoholic fatty liver disease and/or steatohepatitis. Cochrane Database Syst Rev 1: CD004996.

Luedde T, Schwabe RF (2011) NF-kappaB in the liver-linking injury, fibrosis and hepatocellular carcinoma. Nat Rev Gastroenterol Hepatol 8(2): 108-118.

Musso G, Gambino R, Cassader M, Pagano G (2010) A meta-analysis of randomized trials for the treatment of nonalcoholic fatty liver disease. Hepatology 52(1): 79-104.

Pan WH, Wang CY, Huang SM, Yeh SY, Lin WG, Lin DI, Liaw YF (1993) Vitamin A, Vitamin E or beta-carotene status and hepatitis B-related hepatocellular carcinoma. Annals Epidemiol 3(3): 217-224.

Poli G (2000) Pathogenesis of liver fibrosis: role of oxidative stress. Mol Aspects Med 21(3): 49-98.

Qu CX, Kamangar F, Fan JH, Yu B, Sun XD, Taylor PR, Chen BE, Abnet CC, Qiao YL, Mark SD, Dawsey SM (2007) Chemoprevention of primary liver cancer: a randomized, double-blind trial in Linxian, China. J Natl Cancer Inst 99(16): 1240-1247.

Sanyal AJ, Chalasani N, Kowdley KV, McCullough A, Diehl AM, Bass NM, Neuschwander-Tetri BA, Lavine JE, Tonascia J, Unalp A, Van Natta M, Clark J, Brunt EM, Kleiner DE, Hoofnagle JH, Robuck PR (2010) Pioglitazone, vitamin E, or placebo for nonalcoholic steatohepatitis. $N$ Engl J Med 362(18): 1675-1685.

Sanyal AJ, Mofrad PS, Contos MJ, Sargeant C, Luketic VA, Sterling RK, Stravitz RT, Shiffman ML, Clore J, Mills AS (2004) A pilot study of vitamin $\mathrm{E}$ versus vitamin $\mathrm{E}$ and pioglitazone for the treatment of nonalcoholic steatohepatitis. Clin Gastroenterol Hepatol 2(12): 1107-1115.

The ATBC Cancer Prevention Study Group (1994) The alpha-tocopherol, beta-carotene lung cancer prevention study: design, methods, participant characteristics, and compliance. Ann Epidemiol 4(1): 1-10.

Yakaryilmaz F, Guliter S, Savas B, Erdem O, Ersoy R, Erden E, Akyol G, Bozkaya H, Ozenirler S (2007) Effects of vitamin E treatment on peroxisome proliferator-activated receptor-alpha expression and insulin resistance in patients with non-alcoholic steatohepatitis: results of a pilot study. Intern Med J 37(4): 229-235.

Yamamoto Y, Yamashita S, Fujisawa A, Kokura S, Yoshikawa T (1998) Oxidative stress in patients with hepatitis, cirrhosis, and hepatoma evaluated by plasma antioxidants. Biochem Biophys Res Commun 247(1): 166-170.

Yu MW, Chiu YH, Chiang YC, Chen CH, Lee TH, Santella RM, Chern HD, Liaw YF, Chen CJ (1999) Plasma carotenoids, glutathione S-transferase $\mathrm{M} 1$ and T1 genetic polymorphisms, and risk of hepatocellular carcinoma: independent and interactive effects. Am J Epidemiol 149(7): 621-629.

Yu MW, Hsieh HH, Pan WH, Yang CS, CH CJ (1995) Vegetable consumption, serum retinol level, and risk of hepatocellular carcinoma. Cancer Res 55(6): 1301-1305.

Yuan JM, Gao YT, Ong CN, Ross RK, Yu MC (2006) Prediagnostic level of serum retinol in relation to reduced risk of hepatocellular carcinoma. J Natl Cancer Inst 98(7): 482-490.

Zhang W, Shu XO, Li H, Yang G, Cai H, Ji BT, Gao J, Gao YT, Zheng W, Xiang YB (2012) Vitamin intake and liver cancer risk: a report from two cohort studies in china. J Natl Cancer Inst 104(15): 1174-1182.

This work is published under the standard license to publish agreement. After 12 months the work will become freely available and the license terms will switch to a Creative Commons AttributionNonCommercial-Share Alike 3.0 Unported License.

Supplementary Information accompanies this paper on British Journal of Cancer website (http://www.nature.com/bjc) 